\title{
Improving the Efficacy of Multi-objective Evolutionary Algorithms for Real-World Applications (Abstract of Invited Talk)
}

\author{
Kay Chen Tan \\ National University of Singapore \\ 4 Engineering Drive 3, Singapore 117576 \\ eletankc@nus.edu.sg
}

\begin{abstract}
Multi-objective evolutionary algorithms (MOEAs) are a class of stochastic optimization techniques that simulate biological evolution to solve problems with multiple objectives. Multi-objective (MO) optimization is a challenging research topic because it involves the simultaneous optimization of several (and normally conflicting) objectives in the Pareto optimal sense. It requires researchers to address many issues that are unique to MO problems, such as fitness assignment, diversity preservation, balance between exploration and exploitation, elitism and archiving. In this talk, a few advanced features for handling large and computationally intensive real-world MO optimization problems will be presented. These include a distributed cooperative coevolutionary approach to handle large-scale problems via a divide-andconquer strategy by harnessing technological advancements in parallel and distributed systems and a hybridization scheme with local search heuristics for combinatorial optimization with domain knowledge. The talk will also discuss the application of these techniques to various engineering problems including scheduling and system design, which often involve different competing specifications in a large and highly constrained search space.
\end{abstract}

